\title{
ORIGINAL ARTICLE \\ Pretreatment with Lactobacillus reuteri F-9-35 attenuates ethanol-induced gastric injury in rats
}

\author{
Mao-Cheng Sun',2, Ping-Ping Hou', Xin-Yu Wang', Chang-Hui Zhao', Bi-Jun Cheng', \\ Yan-Ling Wang ${ }^{3}$, Hong-Wei Hao ${ }^{4}$, Tie-Hua Zhang ${ }^{*}$, and Hai-Qing Ye ${ }^{\text {** }}$ \\ 'College of Food Science and Engineering, jilin University, Changchun, China; ${ }^{2}$ School of Public Health, Jilin Medical \\ University, jilin City, China; ${ }^{3}$ School of Pharmaceutical Sciences, Jilin University, Changchun, China; ${ }^{4}$ Fullarton \\ Bioengineering Technology Co., Ltd., Beijing, China
}

Abstract

Background: Previous studies suggested that probiotics intervention may be one of the methods for preventing and/or treating gastric ulcer.

Objective: The aim of the study was to compare the preventive effects of a spaceflight mutant Lactobacillus reuteri $\mathrm{F}-9-35$ and its wild type on ethanol-induced gastric injury in rats.

Design: Forty rats were randomly allocated into five groups: a normal group (NOR), ethanol group (EtOH), skim milk group (MILK), L. reuteri F-9-35 group (F935) and wild-type group (WT). The NOR and EtOH groups received $1 \mathrm{ml}$ of distilled water by daily gavage for 14 days. The MILK group received $1 \mathrm{ml}$ of skim milk alone, while the F935 and WT groups were administered $1 \mathrm{ml}$ of skim milk containing the mutant and wild type $\left(1 \times 10^{10}\right.$ colony-forming unit/ml) by daily gavage for 14 days, respectively. Acute gastric injury was induced by absolute alcohol $1 \mathrm{~h}$ after the final administration of different treatments, except for the NOR group. Results: Pretreatment with $L$. reuteri F-9-35, but not milk alone or milk with the L. reuteri wild type, showed significant reduction of ethanol-induced gastric injury, as evidenced by lowering of ulcer index, ulcer area $(\%)$, and histological lesion. F-9-35 decreased the levels of lipid peroxidation and myeloperoxidase and increased mucus, glutathione, and nitric oxide levels in gastric tissue. Moreover, F-9-35 inhibited the expression of proinflammatory genes including gastric tumor necrosis factor- $\alpha$, interleukin- $1 \beta$, and cyclooxygenase- 2 and decreased the activity of nuclear factor kappa B (NF-kB).

Conclusion: These findings indicated that $L$. reuteri $\mathrm{F}-9-35$ pretreatment can attenuate ethanol-induced gastric injury in rats by inhibiting oxidative stress and inflammatory response. Together, L. reuteri F-9-35 has potential preventive efficacy on gastric ulcer.

Keywords: Lactobacillus reuteri; ethanol; gastric injury; inflammatory; antioxidant; NF-кB pathway

Received: 8 June 20 8; Revised: 26 August 20।8; Accepted: 8 September 20।8; Published: 29 October 2018

A lcohol is considered a part of social culture in many countries and is associated with numerous social behaviors, such as party time and celebrations (1). However, the consumption of large amounts of ethanol can directly lead to acute gastric mucosal bleeding, edema, and erosion (2). Long-term drinking has been reported to be associated with gastric mucosal lesions including gastric ulcer and gastritis (3). Although the mechanism of alcoholic gastric damage has not been fully elucidated, accumulating evidence has proved that oxidative stress and inflammatory response are involved in the occurrence and development of gastric mucosal lesions $(4,5)$.

It is well known that probiotics have many health effects on the host when consumed, especially on the gastrointestinal tract (6). Increasing evidence in animal models also suggests that probiotics are promising for preventing and/or treating gastric ulcers. Lactobacillus rhamnosus GG pretreatment attenuates acetic acid-induced gastric ulcer in rats by the regulation of expression of various growth factors (7), as well as ethanol-induced acute gastric mucosa injury by increasing mucosal prostaglandin (PG) E2 level and mucin mRNA expression (8). Treatment of rats with the probiotic mixture VSL \#3 ${ }^{\circledR}$ (eight probiotic bacteria) heals gastric ulcer induced by acetic acid through the inhibition of pro-inflammatory response and the improvement of vascular regenerative protein expression (9). Lactobacillus plantarum LC27 and Bifidobacterium longum LC67 not only alleviate ethanol-induced gastric injury but also ameliorate ethanol-induced hepatic injury in mice (10). 
Lactobacillus reuteri, one species of Lactobacillus, is a candidate for probiotics because it may be able to prevent or alleviate a variety of human diseases, such as early-life disorders, obesity, and enteric infection (11). However, there is no data reported on gastroprotective activity of $L$. reuteri. Therefore, we examined the effect of a spaceflight mutant L. reuteri $\mathrm{F}-9-35$ and its wild type on gastric injury induced by ethanol. The effects of two strains on ulcer index (UI), the percentage of ulcer area (UA), histological lesion, and the levels of malondialdehyde (MDA), myeloperoxidase (MPO), glutathione (GSH), nitric oxide (NO), mucus, and the expression of tumor necrosis factor- $\alpha(\mathrm{TNF}-\alpha)$, interleukin-1 $\beta$ (IL-1 $\beta$ ), cyclooxygenase-2 (COX-2) mRNA, and nuclear factor kappa $\mathrm{B}(\mathrm{NF}-\mathrm{\kappa} \mathrm{B})$ activity in gastric tissue was studied. In addition, hepatic MDA, triglycerides (TG) and GSH levels were also determined.

\section{Materials and methods}

\section{Bacterial strains}

The mutant $L$. reuteri $\mathrm{F}-9-35$ and its wild type $L$. reuteri GS-23 were provided by Fullarton Bioengineering Technology Co., Ltd. (Beijing, China). The wild type was sent into outer space by China's Shenzhou-11 spacecraft in 2016 for 31 days and $18.5 \mathrm{~h}$ of space flight. After the spacecraft landed, one mutant $L$. reuteri F-9-35 was chosen for this study because it had better gastrointestinal resistance and hydrophobicity than its wild type in vitro, and the safety evaluation indicated that the mutant was safe (12).

\section{Preparation of $L$. reuteri for rat feeding}

Two strains were each inoculated in deMan Rogosa and Sharpe (MRS) broth and incubated for $18 \mathrm{~h}$ at $37^{\circ} \mathrm{C}$. The cells were adjusted to a concentration of $1 \times 10^{10}$ colonyforming unit $(\mathrm{CFU}) / \mathrm{mL}$. The pellets were obtained by centrifugation $\left(6,000 \mathrm{~g}, 10 \mathrm{~min}, 4^{\circ} \mathrm{C}\right)$, washed in $0.85 \%$ $\mathrm{NaCl}$ solution twice, and then resuspended in $12 \%(\mathrm{w} / \mathrm{v})$ skim milk sterilized and stored at $-80^{\circ} \mathrm{C}$ until further use.

\section{Animal study design}

Forty female Wistar rats (180-220 g) were obtained from Yisi Experimental Animal Technology Co., Ltd. (Changchun, China). Rats were housed in an air-conditioned room at $22 \pm 2{ }^{\circ} \mathrm{C}$ and had free access to tap water and a standard diet. After acclimation for 7 days, rats were randomly divided into five groups $(n=8)$ : a normal group (NOR), ethanol group (EtOH), skim milk group (MILK), wild type group (WT), and F-9-35 group (F935). The NOR and EtOH groups were administered $1 \mathrm{~mL} /$ day distilled water by daily gavage, while the MILK, WT, and F935 groups received $12 \%$ sterilized skim milk, with or without the wild type or the mutant $\left(1 \times 10^{10} \mathrm{CFU} / \mathrm{mL}\right)$ for 14 days. At Day 13, all rats were fasted but not of water for $24 \mathrm{~h}$ after being gavaged. Then gastric ulcer was induced by intragastric absolute ethanol ( $5 \mathrm{ml} / \mathrm{kg} \mathrm{BW}) 1 \mathrm{~h}$ after the final administration of probiotics $(13,14)$. An hour after ethanol treatment, rats were sacrificed under anesthesia after injection of sodium pentobarbital $(60 \mathrm{mg} /$ $\mathrm{kg} \mathrm{BW}$ ). The stomach was excised and cut open along the greater curvature, cleaned with cold normal saline and macroscopically evaluated for UI scoring as described previously (15). Each stomach was photographed for determination of UA percentage by ImageJ software ( $1.51 \mathrm{j}$, National Institutes of Health, Bethesda, USA). Then each stomach was dichotomized, and $1 \times 1 \mathrm{~cm}$ glandular segments from one moiety was fixed in $10 \%$ neutral formalin buffer for histopathologic analysis; the remaining glandular part was weighed and immersed in $0.1 \%$ Alcian blue 8GX (Yuanye, Shanghai, China) solution for the determination of gastric wall mucus. The other moiety and liver sample were immediately frozen in liquid nitrogen for $3 \mathrm{~h}$ and transferred to $-80^{\circ} \mathrm{C}$ for further determination. The protocol was approved by the Institutional Animal Care and Use Committee of Jilin University.

\section{Histological examination}

Gastric sections fixed by formalin were embedded in paraffin. Full-thickness sections $(5 \mu \mathrm{m})$ were stained with hematoxylin and eosin. The histological damage was evaluated and scored by a pathologist observer in a blinded manner as described previously (16).

\section{Gastric wall mucus determination}

Mucus content was measured by the spectrophotometric method as previously described (17).

\section{Biochemical analysis}

The levels of MPO, MDA, GSH, and NO in gastric tissue and MDA, GSH, and TG in hepatic tissue were determined using commercial kits (Jiancheng Bioengineering Institute, Nanjing, China) according to the manufacturer's instructions. Total protein content was measured with a Bradford Protein Assay Kit (Beyotime, Shanghai, China).

\section{Real-time quantitative PCR}

Real-time quantitative PCR (qPCR) was carried out to assess changes in the expression of mRNA for TNF- $\alpha$, IL-1 $\beta$, and COX-2. Total RNA was extracted from gastric tissue using TRIzol Reagent (Thermo Fisher Scientific, Waltham, USA) according to the manufacturer's protocol. RNA was reverse-transcribed to cDNA with an All-In-One RT 5X MasterMix kit containing a genomic DNA removal procedure (Applied Biological Materials, Richmond, Canada). The qPCR was performed using the EvaGreen 2X qPCR MasterMix (Applied Biological Materials) on a Bio-Rad CFX96 RealTime instrument. The qPCR primers used were as follows: COX-2 forward, GGTTCACCCGAGGACTGGGC, and reverse, CGCAGGTGCTCAGGGACGTG (9); TNF- $\alpha$ 
forward, TGTGCCTCAGCCTCTTCTCATTCA, and reverse, CATTTGGGAACTTCTCCTCCTTG; IL-1 $\beta$ forward, AATGACCTGTTCTTTGAGGCTGAC and reverse, CGAGATGCTGCTGTGAGATTTGAAG; GAPDH forward, TGCTGGTGCTGAGTATGTCGTG, and reverse, CGGAGATGATGACCCTTTTGG (18). The gene expression level was calculated using the $\Delta \Delta \mathrm{Ct}$ method and normalized to GAPDH as an internal control.

\section{Western blot analysis}

The gastric tissues were hand-homogenized in liquid $\mathrm{N}_{2}$ and the total proteins were extracted by a cold lysis buffer with protease and phosphatase inhibitors (Beyotime, Shanghai, China). The protein content was measured using a BCA Protein Assay Kit (Beyotime). Proteins were separated by $12 \%$ sodium dodecyl sulfate-polyacrylamide gels and then transferred onto a PVDF membrane. The membrane was blocked for $2 \mathrm{~h}$ with 3\% bovine serum albumin in TBST buffer (Tris-buffered saline and 0.1\% Tween 20) at room temperature and then incubated overnight with primary antibodies against either $\beta$-actin, NF- $\mathrm{B}$ p-65 (Proteintech, Chicago, USA), or NF-кB p-p65 (Arigo, Hsinchu City, China) at $4^{\circ} \mathrm{C}$ and subsequently with secondary antibody HRP-conjugated goat anti-rat IgG H\&L (Proteintech) for $2 \mathrm{~h}$. Specific bands were detected using a chemiluminescent Western blot imaging system (Azure, Dublin, USA).

\section{Statistical analysis}

Data were presented as mean $\pm \mathrm{SD}$. A Kruskal-Wallis test with Dunn's correct was performed using GraphPad Prism 6.0 (GraphPad Software Inc., San Diego, USA). Statistical significance was set at $p<0.05$.

\section{Results}

\section{Stomach appearances of rats}

The stomach photos after ethanol induction were shown in Fig. 1A through E. The results showed the stomachs from the EtOH group had severe bleeding and ulcer. Although pretreatment with milk alone or two stains relieved gastric damage compared to the EtOH group, the F935 group showed the smallest injury. As shown in Fig. 1F and $\mathrm{G}$, compared with the EtOH group, the UI and UA $(\%)$ in the F935 group were significantly decreased (UI, $26.67 \pm 8.62$ vs. $60.50 \pm 5.47, p<0.01 ; \mathrm{UA}, 10.75 \pm 3.60$ vs. $35.19 \pm 5.21 \%, p<0.01$ ), while there were no significant differences in either the MILK or WT group $(p>0.05)$.

\section{Histological evaluation of gastric injury}

Histological evaluation (Fig. 2) showed the EtOH group (score: $10.33 \pm 1.03$ ) exhibited severe pathological changes including epithelial damage, glandular damage, massive bleeding, and inflammatory cell infiltration. The MILK group (score: $7.67 \pm 1.00$ ) and WT group (score: $6.67 \pm 0.82$ ) showed mild gastric mucosa injury compared to the EtOH group. Notably, minimal damage was observed in the F935 group (score: $2.50 \pm 0.55$ ).

\section{Gastric wall mucus}

The mucus content in different treatment groups is shown in Fig. 3. The mucus content was significantly decreased in the EtOH group compared to the NOR group $(p<0.001)$. Compared with the EtOH group, L. reuteri F-9-35 significantly recovered the mucus content $(p<0.01)$, while animals treated with milk alone or wild type showed no significant difference $(p>0.05)$.

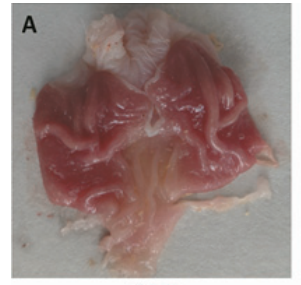

NOR

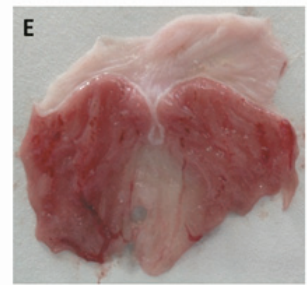

F935

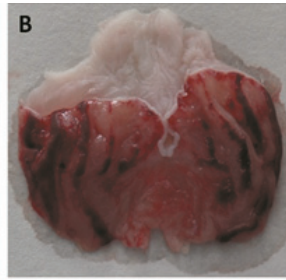

CON

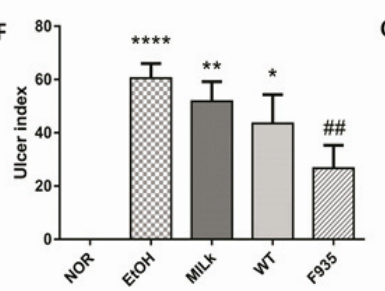

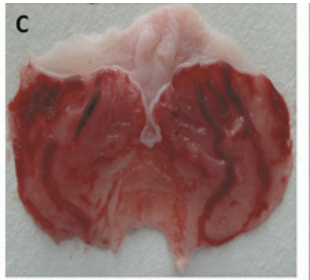

MILK

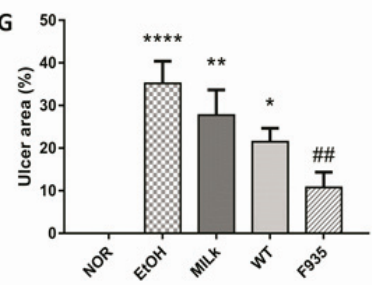

Fig. 1. Macroscopic evaluation of gastric injury. (A) Normal (NOR) group; (B) ethanol (EtOH) group; (C) skim milk (MILK) group; (D) wild-type (WT) group; (E) L. reuteri F-9-35 (F935) group; (F) ulcer index; (G) ulcer area $(\%)$. $* * * p<0.0001$, $* * p<0.01$, and $* p<0.05$ vs. the NOR group; \#\#p<0.01 vs. the EtOH group. 


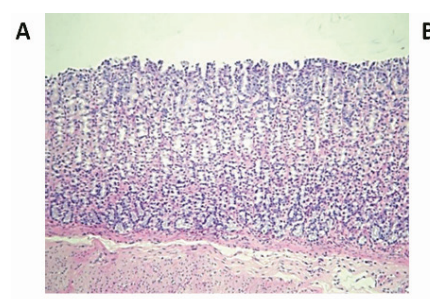

NOR

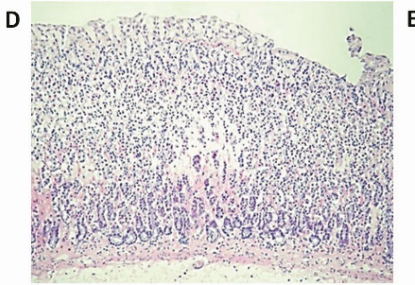

WT

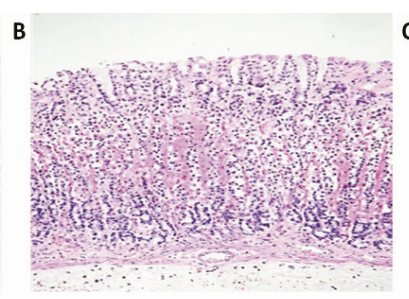

CON

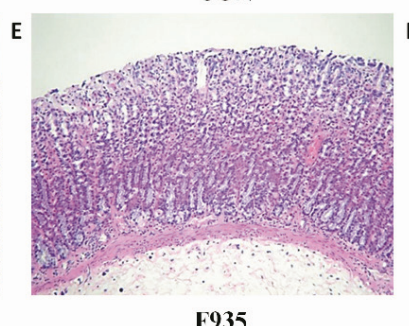

F935

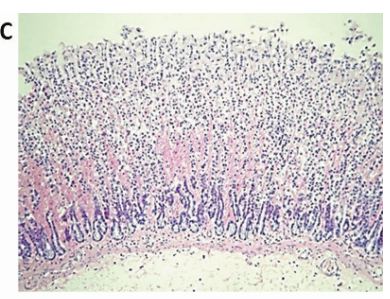

MILK

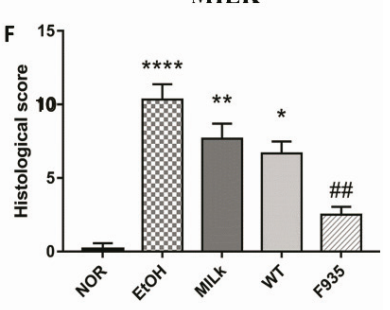

Fig. 2. Histopathological analysis of gastric mucosa (hematoxylin and eosin staining, $\times 100$ ). (A) NOR group; (B) EtOH group; (C) MILK group; (D) WT group; (E) F935 group; (F) histological score. ${ }^{* * *} p<0.0001,{ }^{* *} p<0.01$, and ${ }^{*} p<0.05$ vs. the NOR group, $\# \# p<0.05$ vs. the EtOH group.

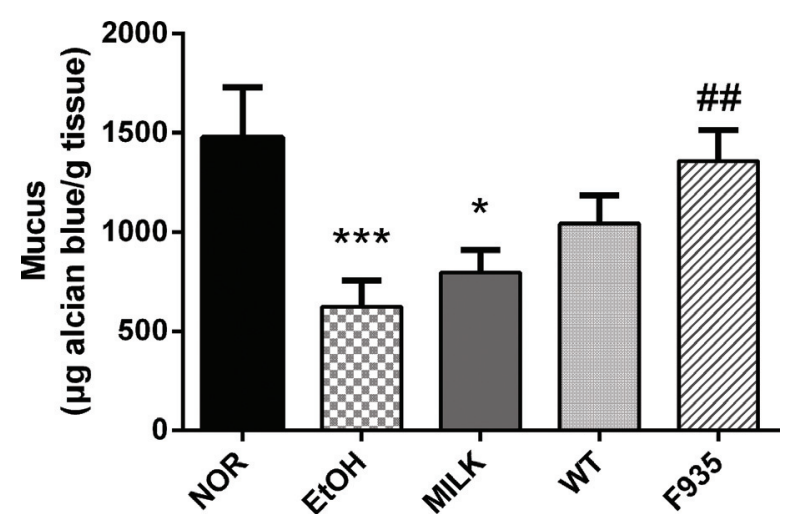

Fig. 3. Effects of $L$. reuteri on gastric wall mucus. ${ }^{* * *} p<0.001$ and $* p<0.01$ vs. the NOR group; \#\#p $<0.01$ vs. the EtOH group.

The levels of MPO, MDA, GSH, and NO in gastric tissue

As shown in Fig. 4, ethanol treatment significantly increased MPO and MDA levels $(p<0.0001)$ and reduced GSH and NO levels $(p<0.0001)$ compared to the NOR group. The GSH, NO, MPO, and MDA levels in the MILK and WT groups had no significant difference compared to the EtOH group $(p>0.05)$. After pretreatment with $L$. reuteri F-9-35, the GSH and NO levels were markedly improved compared to the EtOH group $(p<0.01)$, while the MPO and MDA levels were clearly reduced $(p<0.01)$.

The relative expression of TNF- $\alpha$, IL-I $\beta$, and COX-2 mRNA in gastric tissue

As shown in Fig. 5, the EtOH group showed higher expression of TNF- $\alpha$, IL- $1 \beta$, and COX-2 mRNA (8.79, 5.15, and 6.13 times the NOR group values, respectively). Expression of these genes in the MILK and WT groups decreased, but there were no significant differences compared to the EtOH group ( $p>0.05)$. However, L. reuteri F-9-35 pretreatment significantly suppressed the overexpression of these genes induced by ethanol.

\section{Activation of the NF- $\kappa B$ pathway in gastric tissue}

The ratio of p-p65 to p65 can be used to reflect the activity

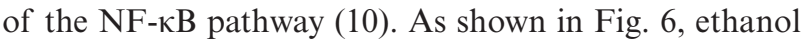
treatment significantly increased $\mathrm{NF}-\kappa \mathrm{B}$ activity in the EtOH, MILK, and WT groups compared to the NOR group ( $p<0.001, p<0.001$, and $p<0.01$, respectively). However, the NF- $\kappa \mathrm{B}$ activity of gastric tissues from F935 rats was significantly downregulated compared to the EtOH group $(p<0.001)$.

\section{Hepatic MDA,TG, and GSH levels}

The MDA and TG levels in the liver had no significant difference among all groups (Fig. 6A and $\mathrm{B}, p>0.05$ ). Of note, the GSH level in the F935 group was significantly higher than that of the NOR or EtOH groups (Fig. 6C; $p<0.01$ and $p<0.05$, respectively).

\section{Discussion}

Exposure of high concentration ethanol in the stomach can result in severe gastric ulcer with bleeding by oxidative stress and inflammatory responses $(19,20)$. Ethanolinduced gastric injury is an important experimental model that is widely used for preclinical evaluation of drugs with potential anti-gastric ulcer activity (21). In this study, we observed that a spaceflight mutant L. reuteri F-9-35 pretreatment markedly attenuated ethanol-induced gastric 
A

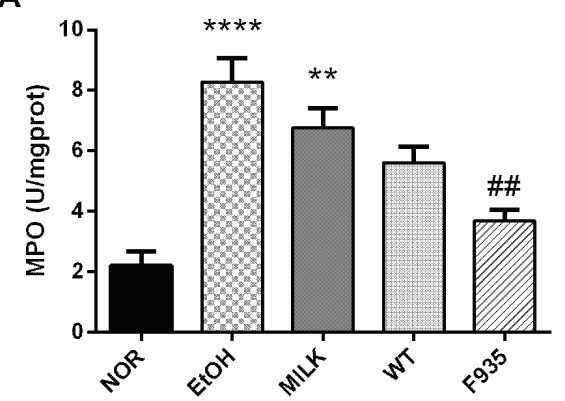

C

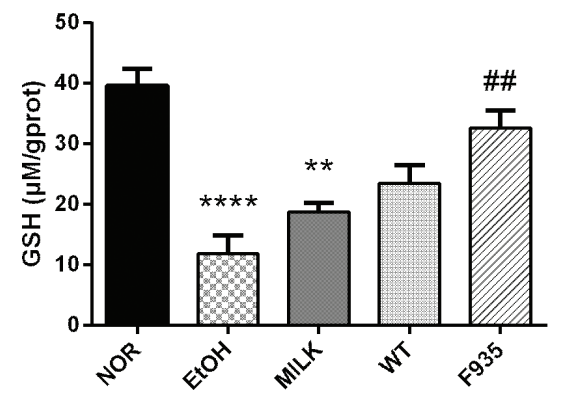

B

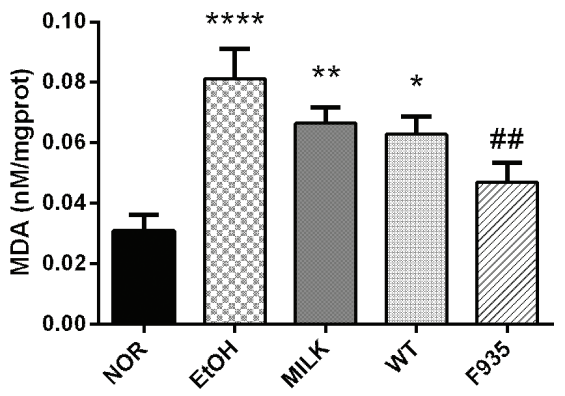

D

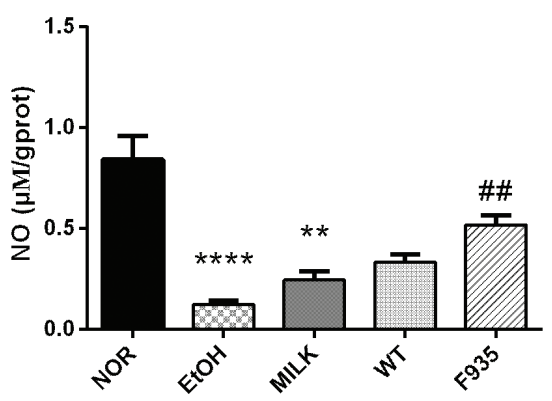

Fig. 4. Effects of L. reuteri on the levels of MPO, MDA, GSH, and NO in gastric tissue. (A) MPO; (B) MDA; (C) GSH; (D) NO. ${ }^{* * * *} p<0.0001,{ }^{* *} p<0.01$, and $* p<0.05$ vs. the NOR group; \#\#p<0.01 vs. the EtOH group. MPO, myeloperoxidase; MDA, malondialdehyde; GHS, glutathione; NO, nitric oxide.
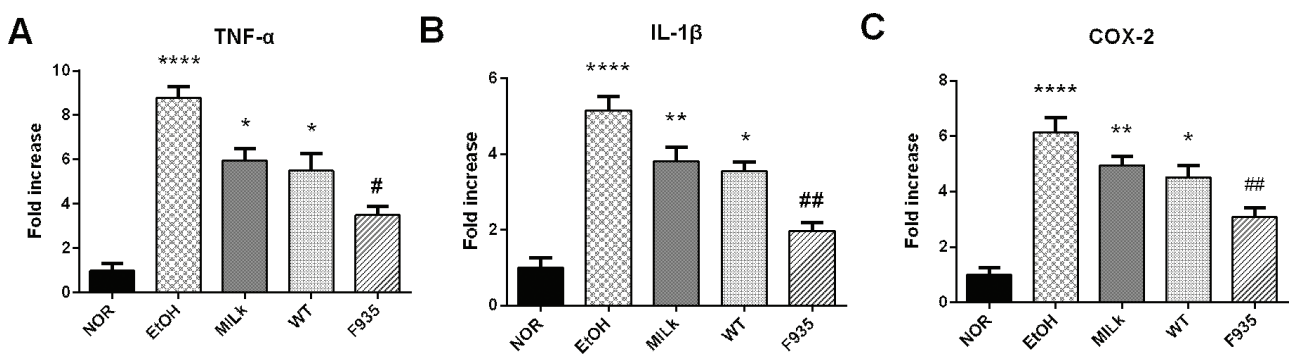

Fig. 5. Effects of $L$. reuteri on the expressions of TNF- $\alpha$, IL-1 $\beta$, and COX-2 mRNA in gastric tissue. (A) TNF- $\alpha$; (B) IL-1 $\beta$; (C) COX-2. ${ }^{* * * *} p<0.0001,{ }^{* *} p<0.01$, and ${ }^{*} p<0.05$ vs. the NOR group; $\# \# p<0.01$ and $\# p<0.05$ vs. the EtOH group. TNF- $\alpha$, tumor necrosis factor- $\alpha$; IL-1 $\beta$, interleukin-1 $\beta$; COX-2, cyclooxygenase- 2 .

injury by inhibiting the major biomarkers of oxidative stress and inflammatory response, but not milk alone or the wild type. The results indicated that the gastroprotective activity of $L$. reuteri was strain-dependent. Our previous study suggested that probiotic characteristics of the mutant were superior to those of its wild type, including gastrointestinal resistance and adhesion (12). These characteristics may help the mutant colonize gastric mucosa after inoculation, and the colonized mutants act as a bacterial barrier to protect the mucosa from ethanol damage. Furthermore, the reduction of Lactobacillus in the gastric mucosa may be a contributing factor for gastric ulcer (22). Collectively, our results suggested that supplementation of Lactobacillus with high colonization ability in gastric mucosa is likely to have anti-gastric ulcer activity.

Mucus is an important defense barrier of the gastric mucosa against injury (23). Ethanol treatment causes mucus depletion of the gastric mucosal tissues (24), which was consistent with our results. In this study, pretreatment with $L$. reuteri $\mathrm{F}-9-35$ can protect the stomach from ethanol damage by inhibiting the depletion of mucus in gastric mucosa, which may be a result of the increased activity of galactosyltransferase, a key enzyme in mucus synthesis (25). Moreover, bioactive NO in the gastric lumen is regarded as a key defense factor that protects the gastric mucosa from noxious agents (26). Decrease 
of gastric blood flow is one cause of gastric mucosa injury induced by ethanol (27). However, NO can inhibit platelet aggregation and thrombosis, enhance the blood flow of gastric mucosa and cell regeneration, and improve mucosal repair (28). A higher level of NO in the stomach from the F935 group was observed in our study, which indicated that $L$. reuteri $\mathrm{F}-9-35$ pretreatment protects gastric mucosa from ethanol damage partly through improving gastric microcirculation.

Oxidative stress plays a significant role in the pathogenesis of gastric injury induced by ethanol (29). Ethanol administration causes the overproduction of reactive oxygen species (ROS) and acceleration of lipid peroxidation to aggravate gastric injury (30). Because ROS could convert unsaturated fatty acids to MDA through lipid
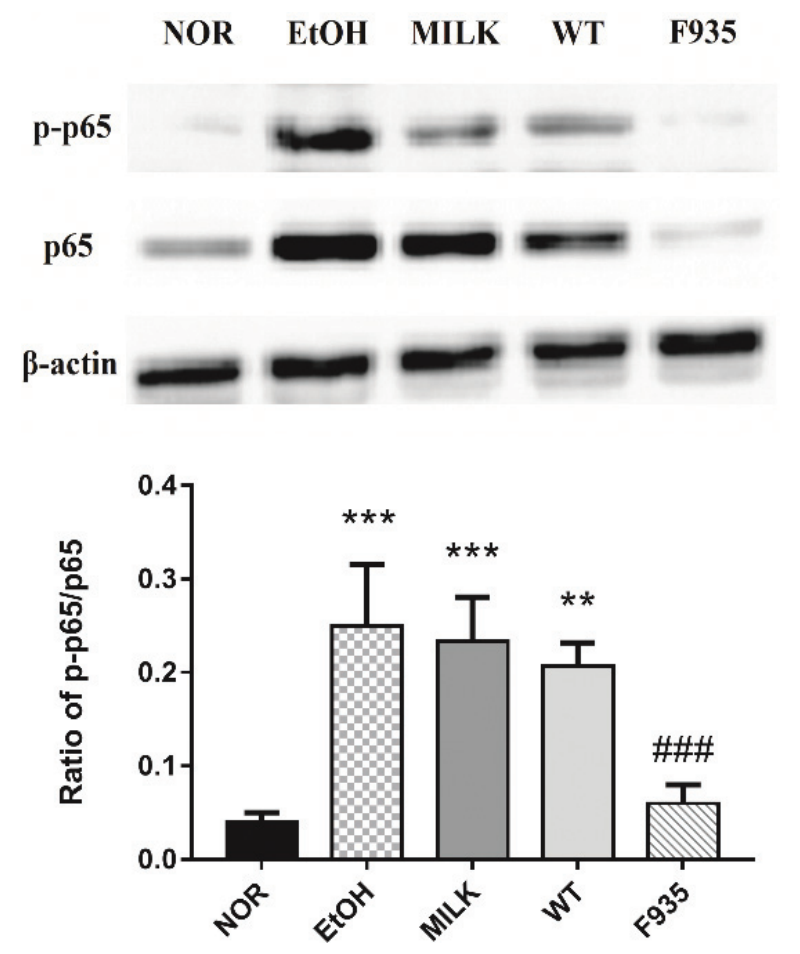

Fig. 6. Effects of L. reuteri on $\mathrm{NF}-\kappa \mathrm{B}$ activity in gastric tissue. $* * * p<0.001$ and $* * p<0.01$ vs. the NOR group; $\# \# \# p<0.001$ vs. the EtOH group. peroxidation, MDA is usually used as a marker of lipid peroxidation (31). GSH is one of the intracellular antioxidants against ROS attacks. Once GSH is depleted, gastric tissue is more vulnerable to ethanol-induced oxidative injury (14). NO has also been proved to be an antioxidant that can accelerate gastric ulcer healing (32). In our study, L. reuteri F-9-35 inhibited MDA increase and improved the reduction of GSH and NO induced by ethanol. These results indicated $L$. reuteri F-9-35 inhibited ethanolinduced oxidative stress, which likely contributes to its gastroprotective action.

Previous studies reported that the higher expressions of some pro-inflammatory genes, such as TNF- $\alpha$, IL-1 $\beta$, and COX-2, were observed in gastric tissue after the intake of ethanol $(33,34)$, which was consistent with our results. TNF- $\alpha$ is a potent pro-inflammatory cytokine that activates neutrophil infiltration to cause the disturbance in gastric microcirculatory, thereby aggravating gastric injury (30). Increased IL-1 $\beta$ could recruit TNF- $\alpha$ and PG in the process of gastric injury induced by ethanol (35). The overexpression of IL- $1 \beta$ and TNF- $\alpha$ in gastric tissue could increase the crucial inflammatory factor COX-2, which converts $\mathrm{PG}$ to oxidation products involved in inflammatory response (36). Additionally, NF- $\kappa \mathrm{B}$ is the classic pro-inflammatory transcription factor, which plays an important role in the inflammatory response of gastric tissue $(34,37)$. Activation of the NF- $\mathrm{kB}$ signaling pathway can induce a large number of inflammatory genes, including TNF- $\alpha$, IL- $1 \beta$, and COX-2 (38). In the present study, pretreatment with $L$. reuteri $\mathrm{F}-9-35$ inhibited the increase of these inflammatory markers and decreased NF- $\mathrm{kB}$ activity. These findings indicated the anti-inflammatory action of L. reuteri F-9-35 against ethanol-induced gastric injury, which may be mediated by the inhibition of NF- $\mathrm{kB}$ activation.

In the present study the mechanism for how $L$. reuteri F-9-35 attenuated ethanol-induced gastric injury in rats was investigated by detecting some biomarkers of oxidative stress and inflammatory response. However, the pathophysiological mechanisms for ethanol-induced gastric ulcer are very complicated. The mechanism may involve gastric mucosal epithelial cell apoptosis, intercellular
A

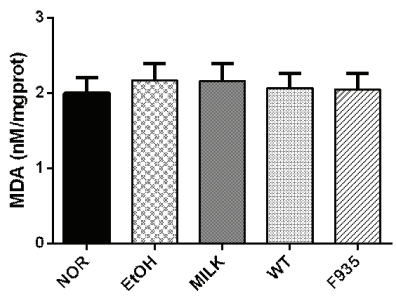

B

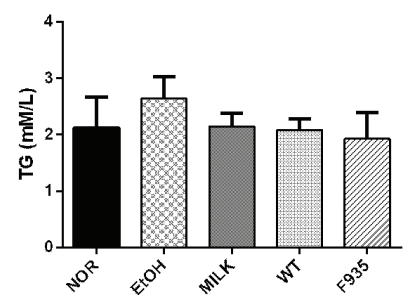

C

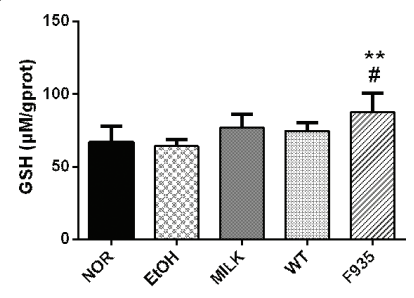

Fig. 7. Effects of L. reuteri on the levels of MDA, TG, and GSH in liver sample. (A) MDA; (B) TG; (C) GSH. ** $p<0.01$ vs. the NOR group; $\# p<0.05$ vs. the EtOH group. MDA, malondialdehyde; TG, triglycerides; GHS, glutathione. 
junction disorders, and alterations in epithelial transport, etc. $(39,40)$. These factors may be potential targets of L. reuteri F-9-35 against gastric ulcer, but further studies are needed. Additionally, it is still uncertain whether it is some metabolites of $L$. reuteri F-9-35 such as exopolysaccharides that contribute to the gastroprotective effect.

Several researches have reported that acute ethanol administration could damage the liver, resulting in dysfunction reflected by increased lipoperoxidation (MDA as a marker) and TG and decreased GSH in animals $(41,42)$. In this study, the levels of hepatic MDA, TG, and GSH in rats were also detected after consumption of ethanol. However, we observed that ethanol treatment did not cause changes in MDA level. Hashimoto and Recknagel (43) reported that ethanol did not increase the hepatic MDA level in rats at any time after binge time, which was consistent with our results. The sole determination of the MDA level is probably not suitable for evaluating hepatic lipid peroxidation in acute ethanol toxicity (44). Additionally, ethanol did not result in elevated TG and decreased GSH in this study, which may be a result of the short alcohol exposure time. Interestingly, pretreatment with L. reuteri F-9-35 enhanced the hepatic GSH level, which indicated the strain may have the potential to protect the liver from oxidative damage. Further study is needed to evaluate the hepatoprotective effect of $L$. reuteri F-9-35 in a model of ethanol-induced liver injury in vivo.

\section{Conclusion}

In conclusion, a spaceflight mutant $L$. reuteri F-9-35 significantly relieved ethanol-induced gastric injury in rats compare to its wild type. This beneficial effect may be a result of a reduction in inflammatory response and oxidative stress by improving mucus secretion and the biosynthesis of GSH and NO, reducing the levels of MPO and MDA, downregulating the expressions of pro-inflammatory genes (TNF- $\alpha$, IL- $1 \beta$, and COX-2 mRNA) and inhibiting NF- $\mathrm{KB}$ activation in gastric tissue. Further efforts are needed to find variations of genome, proteome, and metabolome between L. reuteri F-9-35 and its wild type in order to understand the mechanisms for the different effects of the two strains on anti-gastric ulcer activity.

\section{Acknowledgments}

The authors are thankful to Meng Shi, Xin Su, Yuan Liang, Fu-cheng Zhang, Zheng-zhe Zhang, Qian Li, Hui-qin Liu, Xue Yin, and Shu-ning Zhong, College of Food Science and Engineering, Jilin University, China, for the kind help in intragastric administration, tissue collection, and preparation.

\section{Author contributions}

H.W.H. conceived the study. T.H.Z. and H.Q.Y. designed the experiments. M.C.S., P.P.H., X.Y.W, and B.J.C performed the research. M.C.S. and Y.L.W. analyzed the data. M.C.S and C.H.Z. wrote the paper.

\section{Conflict of interest and funding}

All authors declare that there are no conflicts of interest. This work was supported by the Fullarton Bioengineering Technology Co., Ltd. (Beijing, China) (Project no. FBT2017-001) and the Key Scientific and Technological Projects of Jilin Provincial Science and Technology Department (Project no. 20170204031NY).

\section{References}

1. Heath DB, ed. International handbook on alcohol and culture. Westport: Greenwood Press; 1995.

2. Huh K, Kwon TH, Shin US, Kim WB, Ahn BO, Oh TY, et al. Inhibitory effects of DA-9601 on ethanol-induced gastrohemorrhagic lesions and gastric xanthine oxidase activity in rats. $\mathbf{J}$ Ethnopharmacol 2003; 88(2-3): 269-73.

3. Chou SP. An examination of the alcohol consumption and peptic ulcer association - results of a national survey. Alcohol Clin Exp Res 1994; 18(1): 149-53.

4. Rami AB, Fouad AB, Jamil AMM, Mohammed AA, Hadi HA, Mohd $\mathrm{AH}$, et al. In vivo antioxidant and antiulcer activity of Parkia speciosa ethanolic leaf extract against ethanol-induced gastric ulcer in rats. PLoS One 2013; 8(5): e64751.

5. Asmari AA, Shahrani HA, Masri NA, Faraidi AA, Elfaki I, Arshaduddin M. Vanillin abrogates ethanol induced gastric injury in rats via modulation of gastric secretion, oxidative stress and inflammation. Toxicol Rep 2016; 3: 105-13.

6. Vitetta L, Briskey D, Alford H, Hall S, Coulson S. Probiotics, prebiotics and the gastrointestinal tract in health and disease. Inflammopharmacology 2014; 22(3): 135-54.

7. Lam EK, Yu L, Wong HP, Wu WK, Shin VY, Tai EK, et al. Probiotic Lactobacillus rhamnosus GG enhances gastric ulcer healing in rats. Eur J Pharmacol 2007; 565(1-3): 171-9.

8. Lam EK, Tai EK, Koo MW, Wong HP, Wu WK, Yu L, et al. Enhancement of gastric mucosal integrity by Lactobacillus rhamnosus GG. Life Sci 2007; 80(23): 2128-36.

9. Dharmani P, De Simone C, Chadee K. The probiotic mixture VSL\#3 accelerates gastric ulcer healing by stimulating vascular endothelial growth factor. PLoS One 2013; 8(3): e58671.

10. Kwon EK, Kang GD, Kim WK, Han MJ, Kim DH. Lactobacillus plantarum LC27 and Bifidobacterium longum LC67 simultaneously alleviate ethanol-induced gastritis and hepatic injury in mice. J Funct Foods 2017; 38: 389-98.

11. Mu Q, Tavella VJ, Luo XM. Role of Lactobacillus reuteri in human health and diseases. Front Microbiol 2018; 9: 757.

12. Hao HW, Zhang TH, Sun MC. A space flight-induced mutant L. reuteri F-9-35 and its application. China Patent; No. CN107828703A; 2018.

13. Jin SE, Lee MY, Shin IS, Jeon WY, Ha H. Syzygium aromaticum water extract attenuates ethanolinduced gastric injury through antioxidant effects in rats. Mol Med Rep 2016; 14(1): 361-6.

14. Park SW, Oh TY, Kim YS, Sim H, Park SJ, Jang EJ, et al. Artemisia asiatica extracts protect against ethanol-induced injury in gastric mucosa of rats. J Gastroenterol Hepatol 2008; 23(6): 976-84.

15. Yang J, Zhou W, Gu Y, Dai J, Li X, Tai P, et al. Protective effect of Pu-erh tea extracts against ethanol-induced gastric mucosal damage in rats. Biomedical Rep 2018; 8(4): 335-42. 
16. Jahovic N, Erkanlı G, İșeri S, Arbak S, Alican İ. Gastric protection by $\alpha$-melanocyte-stimulating hormone against ethanol in rats: involvement of somatostatin. Life Sci 2007; 80(11): 1040-5.

17. Corne SJ, Morrissey SM, Woods RJ. Proceedings: a method for the quantitative estimation of gastric barrier mucus. J Physiol 1974; 242(2): 116P-17P.

18. Ali MS, Neisi N, Darbor M, Hassanpour M, Makvandi M, Solgi G. $\beta$-carotene protects the gastric mucosa against ischemia-reperfusion injury in rats. Eur Cytokine Netw 2012; 23(4): 173-8.

19. Konturek PC, Brzozowski T, Pajdo R, Nikiforuk A, Kwiecien S, Harsch I, et al. Ghrelin-a new gastroprotective factor in gastric mucosa. J Physiol Pharmacol 2004; 55(2): 325-36.

20. Tamura M, Matsui H, Kaneko T, Hyodo I. Alcohol is an oxidative stressor for gastric epithelial cells: detection of superoxide in living cells. J Clin Biochem Nutr 2013; 53(2): 75-80.

21. Santos FA, Rao VS. 1,8-cineol, a food flavoring agent, prevents ethanol-induced gastric injury in rats. Dig Dis Sci 2001; 46(2): $331-7$.

22. Almeida LR, Costa PS, Ama N, Reis M, Barros KO, Alvim LB, et al. Porcine stomachs with and without gastric ulcer differ in Lactobacillus load and strain characteristics. Can J Microbiol 2018; 64(7): 493-9.

23. Shorrock CJ, Rees WD. Overview of gastroduodenal mucosal protection. Am J Med 1988; 84(2a): 25-34.

24. Slomiany A, Morita M, Sano S, Piotrowski J, Skrodzka D, Slomiany BL. Effect of ethanol on gastric mucus glycoprotein synthesis, translocation, transport, glycosylation, and secretion. Alcohol Clin Exp Res 1997; 21(3): 417-23.

25. Lindi C, Marciani P, Montorfano G, Omodeosale F. Age-related effects of chronic ethanol intake on physical properties, lipid composition and galactosyltransferase activity of rat small intestine microsomes. Alcohol Alcohol 1996; 31(2): 183-9.

26. Petersson J. Nitrate, nitrite and nitric oxide in gastric mucosal defense. Doctor; Uppsala University, 2008.

27. Yang HJ, Kim MJ, Kwon DY, Kang ES, Kang S, Park S. Gastroprotective actions of Taraxacum coreanum Nakai water extracts in ethanol-induced rat models of acute and chronic gastritis. J Ethnopharmacol 2017; 208: 84-93.

28. Sibilia V, Pagani F, Lattuada N, De LV, Guidobono F, Soglian A, et al. Ticlopidine prevents the formation but delays the healing of ethanol-induced gastric lesions in the rat. Pharmacol Res 2007; 55(5): 418-25.

29. Arab HH, Salama SA, Omar HA, El-Sa A, Maghrabi IA. Diosmin protects against ethanol-induced gastric injury in rats: novel anti-ulcer actions. PLoS One 2015; 10(3): e0122417.

30. Liu Y, Liang J, Wu J, Chen H, Zhang Z, Yang H, et al. Transformation of patchouli alcohol to beta-patchoulene by gastric juice: $\beta$-patchoulene is more effective in preventing ethanol-induced gastric injury. Sci Rep 2017; 7(1): 5591.

31. Ibrahim IAA, Abdulla MA, Hajrezaie M, Bader A, Shahzad $\mathrm{N}, \mathrm{Al}-\mathrm{Ghamdi} \mathrm{SS}$, et al. The gastroprotective effects of hydroalcoholic extract of Monolluma quadrangula against ethanolinduced gastric mucosal injuries in Sprague Dawley rats. Drug Design Dev Ther 2016; 10: 93-105.

32. Konturek SJ, Brzozowski T, Majka J, Pytko-Polonczyk J, Stachura J. Inhibition of nitric oxide synthase delays healing of chronic gastric ulcers. Eur J Pharmacol 1993; 239(1-3): 215-17.
33. Shen Y, Jia S, Chao N, Yu D, Chen Z, Cong W, et al. Mechanistic evaluation of gastroprotective effects of Kangfuxin on ethanol-induced gastric ulcer in mice. Chem Biol Interact 2017; 273: 115-24.

34. Chang X, Luo F, Jiang W, Zhu L, Gao J, He H, et al. Protective activity of salidroside against ethanol-induced gastric ulcer via the MAPK/NF- $\mathrm{KB}$ pathway in vivo and in vitro. Int Immunopharmacol 2015; 28(1): 604-15.

35. Wang QS, Zhu XN, Jiang HL, Wang GF, Cui YL. Protective effects of alginate-chitosan microspheres loaded with alkaloids from Coptis chinensis Franch. and Evodia rutaecarpa (Juss.) Benth. (Zuojin Pill) against ethanol-induced acute gastric mucosal injury in rats. Drug Des Devel Ther 2015; 9: 6151-65.

36. Zhao W, Zhu FW, Fu A, Zheng L, Yan Z, Zhao L, et al. Protective effects of DIDS against ethanol-induced gastric mucosal injury in rats. Acta Biochim Biophys Sin 2009; 41(4): 301-8.

37. Keshk WA, Zahran SM, Katary MA, Da EAA. Modulatory effect of silymarin on nuclear factor-erythroid-2-related factor 2 regulated redox status, nuclear factor- $\kappa \mathrm{B}$ mediated inflammation and apoptosis in experimental gastric ulcer. Chem Biol Interact 2017; 273: 266-72.

38. Piao X, Li S, Sui L, Guo L, Liu X, Li H, et al. 1-Deoxynojirimycin (DNJ) Ameliorates indomethacin-induced gastric ulcer in mice by affecting NF-kappaB signaling pathway. Front Pharmacol 2018; 9: 372.

39. Li JH, Ju GX, Jiang JL, Li NS, Peng J, Luo XJ. Lipoic acid protects gastric mucosa from ethanol-induced injury in rat through a mechanism involving aldehyde dehydrogenase 2 activation. Alcohol 2016; 56: 21-8.

40. Luo XJ, Liu B, Dai Z, Li TB, Li NS, Zhang XJ, et al. Expression of apoptosis-associated microRNAs in ethanol-induced acute gastric mucosal injury via JNK pathway. Alcohol 2013; 47(6): 481-93.

41. Wang Y, Liu Y, Sidhu A, Ma Z, Mcclain C, Feng W. Lactobacillus rhamnosus GG culture supernatant ameliorates acute alcohol-induced intestinal permeability and liver injury. Am J Physiol Gastrointest Liver Physiol 2012; 303(1): G32-41.

42. Videla LA, Fernandez V, Ugarte G, Valenzuela A. Effect of acute ethanol intoxication on the content of reduced glutathione of the liver in relation to its lipoperoxidative capacity in the rat. FEBS Lett 1980; 111(1): 6-10.

43. Hashimoto S, Recknagel RO. No chemical evidence of hepatic lipid peroxidation in acute ethanol toxicity. Exp Mol Pathol 1968; 8(2): 225-42.

44. Nordmann R, Ribiere C, Rouach H. Implication of free radical mechanisms in ethanol-induced cellular injury. Free Radic Biol Med 1992; 12(3): 219-40.

\section{*Tie-Hua Zhang \\ No. 5333, Xi'an Rd. \\ Changchun 130062, China. \\ Email: zhangth@jlu.edu.cn}

\section{*Hai-Qing Ye}

No. 5333, Xi'an Rd.

Changchun 130062, China.

Email:yehq@jlu.edu.cn 